\title{
Changes in space weather and heliospheric oscillations due to rotation and rearrangement of solar magnetic field
}

\author{
A.V. Mordvinov, L.A. Plyusnina and V.V. Pipin \\ Institute of Solar-Terrestrial Physics, P.O. Box 4026, Irkutsk, Russia email: avm@iszf.irk.ru
}

Based on analyzing the fine structure of time series of solar and heliospheric parameters a universal numerical technique is developed to study large-scale patterns of solar activity and the heliospheric structure. The Solar Mean Magnetic Field (SMMF) time series and the magnetic fields in solar corona (Hoeksema \& Scherrer 2004) were studied in time-longitude aspect (figures 1a,b,c,d). It is shown that the behavior of the largescale magnetic field, its multiperiodic rotation determine the heliospheric structure and changes in space weather (Mordvinov \& Plyusnina 2004). Time-longitude analysis of Interplanetary Magnetic Field (IMF) near the Earth (King et al. 2004) reveals similar diagram (figure 1e) shifted relative to the photospheric and coronal magnetic fields by the transport time.

The time-longitude behavior of both the flare ensemble and Solar Proton Events (SPEs) demonstrate their complicated synergy governed by the large-scale magnetic field, its rearrangements and changes in the rotational regime. Sources of solar cosmic rays are also associated with long-lived patterns of flaring activity. Figure 1(f) shows a comparative time-longitude analysis that demonstrates changes in space weather due to rearrangements of large-scale magnetic field of the Sun for 1975-2003 (Mordvinov et al. 2002). The major SPEs tend to occur near the abrupt restructuring of large-scale magnetic field.

A study of the rotational modulation of solar and heliospheric parameters reveals cyclic changes in their rotation. Modes of slow rotation dominate at the phase of activity rise whereas the fast modes dominate at the phase of descending activity (Mordvinov \& Plyusnina 2000). The alternating rotational modes appear also in the heliosphere. The time-longitude analysis of the IMF time series provides a comprehensive picture of solar rotation and reveals the $11 / 22$-yr modulation of the heliospheric parameters for $1926-$ 2003. These cyclic changes are interpreted as manifestation of solar torsional oscillations in the heliosphere.

Persistent rotational modes appear also in the IMF time-longitude diagram on a secular timescale. Numerical models describe the secular changes in solar rotation due to magnetic modulation of the angular momentum transport in the convection zone of the Sun (Pipin 2004).

This research is supported by the RFBR 02-02-16044 and INTAS 2001-0550.

\section{References}

Hoeksema, J.T. \& Scherrer, P.H. 2004 http://sun.stanford.edu

King et al. 2004 http://nssdc.gsfc.nasa.gov/omniweb

Mordvinov, A.V. \& Plyusnina, L.A. 2000 Solar Phys. 197, 1.

Mordvinov, A.V. \& Plyusnina, L.A. 2004 Solar-Terrestrial Physics 4, 49.

Mordvinov, A.V., Salakhutdinova, I.I., Plyusnina, L.A., Makarenko, N.N., and Karimova, L.M. 2002 Solar Phys. 211, 241.

Pipin, V.V. 2004 Astronomy Reports 48, 418. 

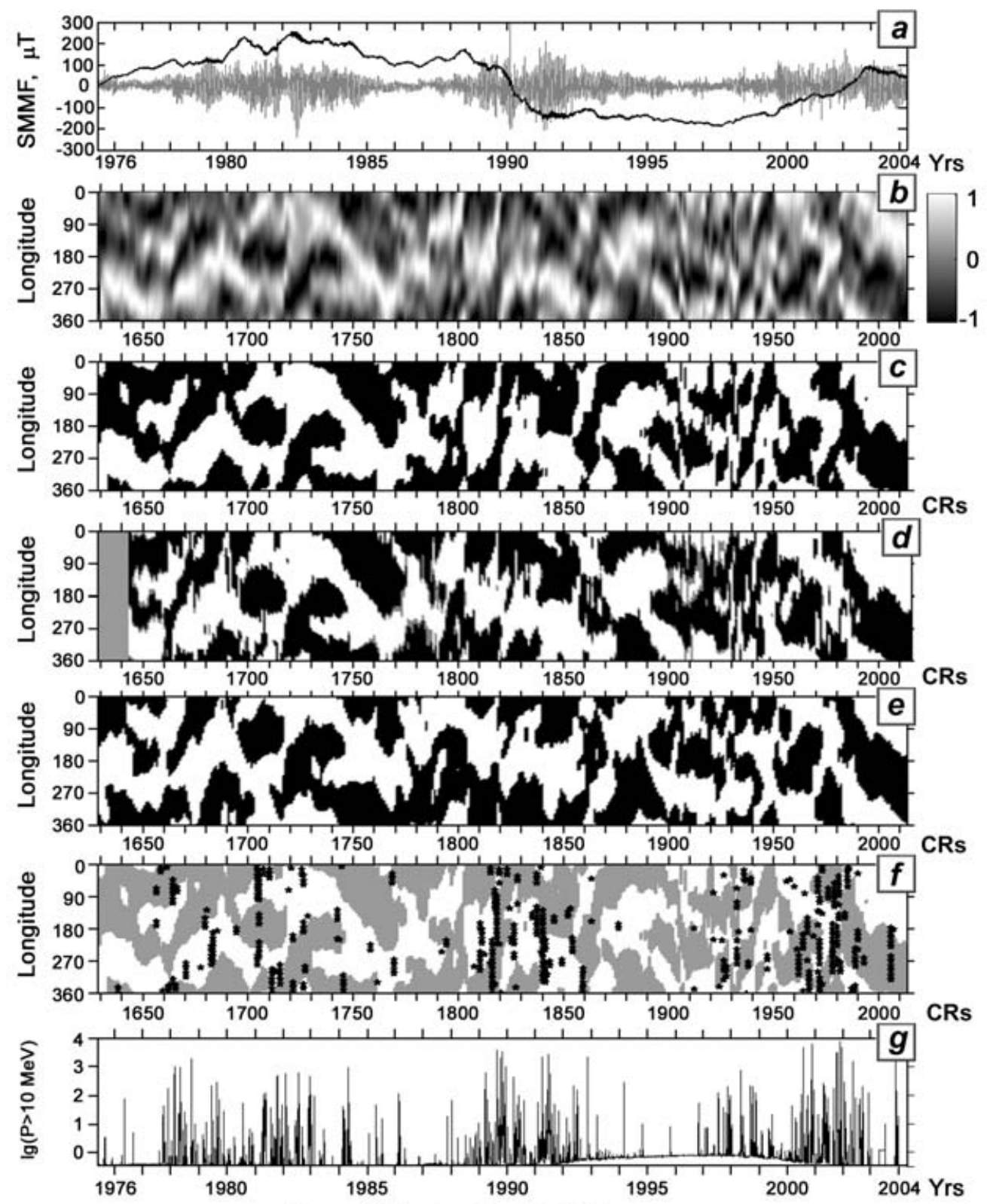

Years / Carrington Rotations

Figure 1. The SMMF and its cumulative sum plots (a), the time-longitude diagram (b), the SMMF diagram indicating magnetic polarities (c); the diagram compiled of the distributions of magnetic polarities in the equatorial profiles of the synoptic maps of extrapolated magnetic fields (d); the time-longitude diagram of the IMF (e); black marks indicate SPEs $>10 \mathrm{MeV}$ exceeding the threshold of $10\left(\mathrm{~cm}^{2} \mathrm{sec} \cdot \mathrm{ster}\right)^{-1}$ superimposed on the SMMF diagram (f), and the plot of the proton flux $>10 \mathrm{MeV}(\mathrm{g})$ 